\title{
The Management of Chronic Myeloid Leukemia in the Era of Second Generation Tyrosine Kinase Inhibitors
}

\author{
Ibrahim C. HAZNEDAROGLU ${ }^{1}$, Mustafa CETINER ${ }^{2}$, Osman ILHAN ${ }^{3}$ \\ ${ }^{1}$ Hacettepe University Faculty of Medicine, Department of Hematology, Ankara \\ ${ }^{2}$ American Hospital Department of Hematology, Istanbul \\ ${ }^{3}$ Ankara University Faculty of Medicine, Department of Hematology, Ankara, TURKEY
}

\begin{abstract}
Chronic Myeloid Leukemia (CML) is a clonal myeloproliferative disorder of pluripotent hematopoietic stem cells, characterized by increased proliferation, and decreased apoptosis of myeloid progenitor cells ${ }^{1}$ with peripheral leukokocytosis. ${ }^{2} \mathrm{CML}$ is a malignant disorder of the stem cell due to reciprocal balanced translocation of genetic material between the long arms of chromosomes 9 and $22 \mathrm{t}(9 ; 22)(\mathrm{q} 34 ; \mathrm{q} 11){ }^{3}$. The shortened chromosome 22 can be visualized by standard cytogenetic techniques and was termed as Philadelphia Chromosome. Fibrosis and biological abnormalities of cytokine network may be evident during the disease course. ${ }^{47}$ The treatment agenda for CML, from past history to today, includes cytotoxic chemoterapy, $\alpha$-interferon (alpha-IFN), allogeneic hematopoietic stem cell transplantation (allo$\mathrm{SCT})^{8}$, imatinib mesylate and second generation tyrosine kinase inhibitors (TKIs) dasatinib and nilotinib..$^{13,8-10}$ Failure to proper management of CML may result in blastic crisis, which can be "difficult-to-manage" with only combination chemotherapy and dasatinib. ${ }^{1,11,12}$
\end{abstract}

Imatinib which is introduction of the first TKI, into clinical practice after IRIS trial ${ }^{13}$, has dramatically changed treatment and follow-up of CML. ${ }^{1,13}$ TKIs specifically targets tyrosine kinase activity of the oncogenic protein encoded by bcr/abl gene. Currently, two more powerful second generation TKIs are available for clinical management of CML, namely dasatinib ${ }^{14}$ and nilotinib. ${ }^{15}$ The European LeukemiaNet (ELN) 2009 recommendations have placed second generation TKIs for the second-line treatment of imatinib-intolerant or imatinib-resistant CML. ${ }^{16,17}$ However, two recently published Phase III randomized clinical trials, Dasatinib Versus Imatinib Study in Treatment-Naive CML Patients (DASISION) ${ }^{14}$ and Evaluating Nilotinib Efficacy and Safety in Clinical Trials-Newly Diagnosed Patients (ENESTnd) ${ }^{15}$ have led to the FDA and EMEA 2011 approval of second generation TKIs for the first-line management of newly diagnosed CML. Dasatinib and nilotinib has shown superior results, such as earlier cytogenetic response and a deeper and durable molecular response in comparison to imatinib in the DASISION ${ }^{14}$ and ENESTnd ${ }^{15}$ trials.

Recent findings raised the criticism about ELN 2009 recommendations regarding the preservation of the ambiguous concept "suboptimal response to imatinib". ${ }^{9.18}$ This gray-zone concept has been generated without long-term follow-up data on imatinib and in the absence of second generation TKIs. ${ }^{16}$ 
Table 1. Summary of Suggested Practical Guidelines by MD Anderson Cancer Center During Chronic Phase Chronic Myeloid Leukemia Therapy ${ }^{20}$

Achievement of CCyR remains the most important goal of therapy; achievement of MMR may be additionally beneficial but should not guide therapy decisions

Molecular monitoring as a single tool after achieving MMR in CCyR is reasonable but over-reaction to modest changes should be avoided; in CCyR (with or without MMR), monitoring with peripheral molecular and FISH studies ensures robust interpretation of response results

In stable CCyR (usually after 2 years of therapy) sudden blastic transformations are rare; consequently, monitoring response every 6 months is reasonable; routine blood tests and physician visits every 3 months help emphasize/monitor patient compliance

In stable CCyR, routine marrow studies are not necessary (replaced by peripheral molecular and FISH studies); bone marrow cytogenetics indicated if there is evidence of chromosomal abnormalities in Ph negative cells (then perform marrow cytogenetics every 6 months until disappearance of abnormalities), at the time new therapies are started, and in case of unexplained cytopenias or significant changes in peripheral blood differential (e.g., increase in blasts or basophils)

Imatinib blood levels is not a useful monitoring procedure

Mutational studies at the time of cytogenetic or hematologic relapse are useful in guiding the choice of the next therapy

Abbreviations: CCyR, complete cytogenetic response; MMR, major molecular response; FISH, fluorescent in situ hybridization studies; Ph-negative, Philadelphia chromosome-negative ${ }^{20}$.

Based on the ELN 2009 recommendations; "Continue imatinib same dose; or test high dose imatinib..." was suggested as a treatment for "imatinibsuboptimal responders". High dose imatinib, which had inferior efficacy when compared to dasatinib and nilotinib, is not superior to standard dose imatinib too. ${ }^{19}$ Increased ratio of complete cytogenetic response (CCyR) and faster, earlier, deeper, durable, more common sustained major molecular response (MMR) obtained via second generation TKIs prompted the earlier administration of dasatinib or nilotinib during the clinical course of CML. More powerful TKIs shall be given at the earlier $\operatorname{sign}(\mathrm{s})$ of resistance and/or intolerance to frontline imatinib. ${ }^{20}$ The elucidation of the superiority of frontline dasatinib or nilotinib could be possible with the upcoming three-year follow-up of the DASISION ${ }^{14}$ and ENESTnd ${ }^{15}$ trials.

Current optimal targets based on ELN 2009 in a chronic phase $(\mathrm{CF}) \mathrm{CML}$ patient receiving imatinib is the occurrence of complete hematological remission (CHR) within 3 months, partial cytogenetic response (PCyR) at 6 months, $\mathrm{CCyR}$ at 12 months, and MMR at 18 months. ${ }^{8}$ More powerful TKIs, dasatinib and nilotinib should be administered at the earlier stage if imatinib fails to produce those responses in these critical time points..$^{20}$ On the other hand, if second generation TKIs would be the frontline treatment for CF- CML patient, the ideals could be CHR within 3 months, CCyR at 6 months, MMR at 12 months, and CMR at 24 months. However, the follow-up period of frontline dasatinib and nilotinib studies are relatively short. Therefore, presently in 2011 the most practical follow-up guidelines have been established by Kantarjian and Cortes from MD Anderson Cancer Center. ${ }^{20}$ Their recommendations are depicted in Table 1. Today, the best way for treatment of CF CML is tyrosine kinase inhibition and the best follow-up of the CML patient with appropriate essential interventions.

In this supplementary issue of UHOD, the selections of second generation TKIs ${ }^{21}$, their long-term adverse effects with proper follow-up and management $^{22}$, treatment of advanced-phase CML with focus on transplantation ${ }^{23}$, and current problems in 
the management of chronic myeloid leukemia in Turke ${ }^{24}$ have been summarized. Near future holds promise for the better CML management, operational cure of CML, or even cure of the disease with the discontinuation of TKI and/or targeting CML stem cell.

\section{REFERENCES}

1. Buyukasik Y, Haznedaroglu IC, Ilhan O, et al. Chronic Myeloid Leukemia: Practical Issues in Diagnosis, Treatment and Follow-Up. UHOD 20 [Suppl 1]: 1-12, 2010.

2. Hayran M, Koca E, Haznedaroglu IC, et al. Predicting chronic leukaemias from assessment of complete peripheral blood counts. J Int Med Res 34: 640-647, 2006.

3. Koca E, Haznedaroglu IC. Imatinib mesylate and the management of chronic myeloid leukemia (CML). Turk J Haematol 22: 161-172, 2005.

4. Cobankara V, Oran B, Ozatli D, et al. Cytokines, endothelium, and adhesive molecules in pathologic thrombopoiesis. Clin Appl Thromb Hemost 7: 126130, 2001.

5. Sayinalp N, Cinar H, Uner A, et al. Plasma basic fibroblast growth factor and bone marrow fibrosis in clonal myeloproliferative disorders. Clin Lab Haematol 26: 265-268, 2004.

6. Akdogan E, Sonmez M, Yilmaz M, et al. The development of chronic myelogenous leukemia in a patient with malign fibrous histiositom treated with radiotherapy: Case report. UHOD 18: 116-120, 2008.

7. Ozatli D, Timuragaoglu A, Alanoglu G, et al. BCR-ABL transcript level and neutrophil alkaline phosphatase activity in CML patients treated with imatinib. UHOD 20: 163-168, 2010.

8. Koca E, Halil M, Adibelli Z, et al. Management of chronic myeloid leukemia (CML) in the pre-imatinib and imatinib era. Haema 9: 301-303, 2006.

9. Beyazit Y, Kekilli M, Haznedaroglu IC. Second-generation BCR-ABL kinase inhibitors in CML. N Engl J Med 363: 1673-1673, 2010.

10. Saydam G, Haznedaroglu IC, Temiz Y, et al. Retrospective evaluation of patients treated with dasatinib for philadelphia positive leukemias: Turkish experience of 16 months. UHOD 19: 195-204, 2009.

11. Bektas $O$, Haznedaroglu IC, Buyukasik $Y$, et al. Dasatinib-induced tumor lysis syndrome and following hematologic remission in fibrotic blastic crisis of CML. UHOD 20 [Suppl 1]: 13-16, 2010.

12. Kaygusuz G, Kuzu I, Akpinar E, et al. Extramedullary hematopoiesis in the axillary lymph node in a patient with an accelerated phase of chronic myeloid leukemia. Turkish J Hematology 26: 40-41, 2009.

13. O'Brien SG, Guilhot F, Larson RA, et al. Imatinib compared with interferon and low-dose cytarabine for newly diagnosed chronic-phase chronic myeloid le- ukemia. N Engl J Med 348: 994-1004, 2003.

14. Kantarjian H, Shah NP, Hochhaus A, et al. Dasatinib versus imatinib in newly diagnosed chronic-phase chronic myeloid leukemia. N Engl J Med 362: 22602270, 2010.

15. Saglio G, Kim DW, Issaragrisil S, et al. Nilotinib versus imatinib for newly diagnosed chronic myeloid leukemia. N Engl J Med 362: 2251-2259, 2010.

16. Baccarani M, Cortes J, Pane F, et al. Chronic myeloid leukemia: an update of concepts and management recommendations of European LeukemiaNet. J Clin Oncol 27: 6041-6051, 2009.

17. Talpaz M, Shah NP, Kantarjian H, et al. Dasatinib in imatinib-resistant Philadelphia chromosome-positive leukemias. N Engl J Med 354: 2531-2541, 2006.

18. Haznedaroglu IC, Koca E, Aksu S, et al. The gray-zone concept, suboptimal response to Imatinib, shall be removed from the ELN-CML recommendations. UHOD 20 [Suppl 1]: 25-26, 2010.

19. Baccarani M, Rosti G, Castagnetti F, Haznedaroglu IC et al. Comparison of imatinib $400 \mathrm{mg}$ and $800 \mathrm{mg}$ daily in the front-line treatment of high-risk, Philadelphiapositive chronic myeloid leukemia: A European LeukemiaNet Study. Blood 113: 4497-4504, 2009.

20. Kantarjian H, Cortes J. Considerations in the management of patients with philadelphia chromosome positive chronic myeloid leukemia receiving tyrosine kinase inhibitor therapy. J Clin Oncol 29: 1512-1516, 2011.

21. Buyukasik Y. Use of second generation tyrosine kinase inhibitors for second-line treatment of chronic myeloid leukemia after imatinib failure. UHOD 21 [Suppl 2]: 4-9, 2011.

22. Yavuz AS. Long-term treatment of chronic myeloid leukemia. UHOD 21 [Suppl 2]: 10-17, 2011.

23. Gulbas Z. Advanced-phase chronic myeloid leukemia treatment and transplantation. UHOD 21 [Suppl 2]: 18-23, 2011.

24. Demirkan F. Current problems in the management of chronic myeloid leukemia in Turkey. UHOD 21 [Suppl 2]: 24-28, 2011.

\section{Correspondence}

Dr. İbrahim C. HAZNEDAROĞLU

Hacettepe Üniversitesi Erişkin Hastaneleri

Hematoloji Ünitesi

06100 Sinhiye, Ankara / TURKEY

Tel: (+90 312) 3051543

Fax: (+90 312) 3051614

e-mail: haznedar@yahoo.com 